\title{
Long non-coding RNA LINC01419 mediates miR-519a-3p/PDRG1 axis to promote cell progression in osteosarcoma
}

Zhiqian $\mathrm{Gu}^{1,2}$, Shaokun $\mathrm{Wu}^{1,2^{*}}$, Jingnan Wang ${ }^{1,2}$ and Shoujun Zhao ${ }^{1,2}$

\begin{abstract}
Background: Osteosarcoma (OS) is one of the most aggressive malignancies with mortality rate worldwide. Accumulating evidence has revealed that long noncoding RNAs (IncRNAs) exert important functions in regulation of cancer initiation and progression. Recently, long intergenic non-protein coding RNA 1419 (LINC01419) has been reported to function as an oncogene in several cancers. However, its role in OS has not been explored yet.

Methods: qRT-PCR and western blot analyses were implemented to determine the expression of genes. The function of OS cells was assessed through colony formation, EdU, JC-1, TUNEL, transwell, and immunofluorescence (IF) assays. FISH and subcellular fractionation assays were conducted to estimate the localization of LINC01419 in OS cells. The interaction between genes was validated through luciferase reporter and RNA pull down assays.

Results: LINC01419 expression was elevated in OS tissues and cells. Functionally, LINC01419 accelerated OS cell proliferation, motility and EMT. In vivo assay showed that silencing LINC01419 hindered the growth of OS tumors. Mechanistic investigation unveiled that LINC01419 acted as a competing endogenous RNA (ceRNA) to augment PDRG1 expression by miR-519a-3p sequestration. Rescue assays verified the oncogenic effect of LINC01419/miR519a-3p/PDRG1 axis on OS development.
\end{abstract}

Conclusion: LINC01419 mediates malignant phenotypes in OS by targeting miR-519a-3p/PDRG1 axis.

Keywords: LINC01419, miR-519a-3p, PDRG1, Osteosarcoma

\section{Background}

Osteosarcoma (OS), a commonly diagnosed malignancy in skeletal system, mainly occurs in adolescents or children and affects the bone growth [1]. In pediatric bone malignancies, OS occupies about $5 \%$ of pediatric cancers [1]. In recent years, the 5-year survival rate of OS patients has been continuously improved because of the introduction of resection surgery and chemoradiotherapy [2]. Nevertheless, obtained chemoresistance and

\footnotetext{
*Correspondence: Shao407370036@163.com

${ }^{1}$ Department of Orthopedics, Hwa Mei Hospital, University of Chinese Academy of Sciences (Ningbo NO. 2 Hospital), No. 41 Northwest Street, Ningbo 315010, Zhejiang, China

Full list of author information is available at the end of the article
}

high recurrence rate caused by distant metastasis significantly reduced the survival rate of most OS patients [3, 4]. Therefore, it is urgent for us to explore new molecular mechanisms underlying the pathogenesis and progression of OS.

Recent studies have demonstrated that over $98 \%$ of human transcriptome lacking protein-coding ability are recognized as non-coding RNAs (ncRNAs) [5]. In addition, ncRNAs comprise long non-coding RNAs (lncRNAs) and microRNAs (miRNAs) [6-8]. LncRNAs are a group of ncRNAs with more than 200 nucleotides in length [9]. Accumulating studies demonstrated that lncRNAs could function in many cancers to affect the progression of cancers, such as epithelial ovarian cancer, papillary thyroid cancer and gastric cancer [10-12]. It

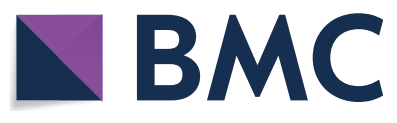

c) The Author(s) 2020. This article is licensed under a Creative Commons Attribution 4.0 International License, which permits use, sharing, adaptation, distribution and reproduction in any medium or format, as long as you give appropriate credit to the original author(s) and the source, provide a link to the Creative Commons licence, and indicate if changes were made. The images or other third party material in this article are included in the article's Creative Commons licence, unless indicated otherwise in a credit line to the material. If material is not included in the article's Creative Commons licence and your intended use is not permitted by statutory regulation or exceeds the permitted use, you will need to obtain permission directly from the copyright holder. To view a copy of this licence, visit http://creativeco mmons.org/licenses/by/4.0/. The Creative Commons Public Domain Dedication waiver (http://creativecommons.org/publicdomain/ zero/1.0/) applies to the data made available in this article, unless otherwise stated in a credit line to the data. 
has been proved that lncRNAs that are aberrantly regulated in cancer could modulate diverse biological behaviors, such as cell proliferation, apoptosis, migration and epithelial-mesenchymal transition (EMT) [13, 14]. In mechanisms, lncRNAs could regulate gene expression through gene imprinting or epigenetic modification. Besides, increasing studies proposed that lncRNA could function as a competing endogenous RNA (ceRNA) by sponging miRNAs to elevate target gene expression. For instance, lncRNA PVT1 sponged miR-424-5p to upregulate CARM1 and then modulate the development of non-small-cell lung cancer [15]. LncRNA SNHG5 regulated miR-26a-5p/TRPC3 axis to facilitate cell growth and invasion in melanoma [16]. Long intergenic non-protein coding RNA 1419 (LINC01419) has been demonstrated as an oncogene in lung adenocarcinoma, esophageal squamous cell carcinoma and gastric cancer [17-19]. However, its role and possible molecular mechanism have not been studied in OS yet.

In this study, we probed into the function of LINC01419 in OS and found the high expression trend and tumor-facilitating role of LINC01419 in OS, providing a new thought for future OS treatment.

\section{Methods}

\section{Tissue samples}

The OS tissues $(n=50)$ and adjacent non-tumor tissues $(n=50)$ were collected from September 2012 to August 2017 in the Hwa Mei Hospital, University of Chinese Academy of Sciences (Ningbo NO. 2 Hospital). All patients accepted no any therapy before surgery and have signed informed consent prior to this study. Samples were sharply frozen in liquid nitrogen and then preserved in $-80^{\circ} \mathrm{C}$. This study was permitted and approved by the Ethics Committee of Hwa Mei Hospital, University of Chinese Academy of Sciences (Ningbo NO. 2 Hospital).

\section{Cell lines}

Human OS cell lines (143B, U2OS, Saos-2) and normal osteoblast (hFOB1.19) were commercially acquired from ATCC (Rockville, Maryland). Saos-2 was maintained in DMEM (Invitrogen, Carlsbad, CA) with 15\% FBS (Gibco, Grand Island, NY) and 1\% antibiotics, whereas other cells were grown in DMEM (Invitrogen) with 10\% FBS (Gibco) and $1 \%$ antibiotics. All cells were incubated under $5 \%$ $\mathrm{CO}_{2}$ at $37^{\circ} \mathrm{C}$.

\section{RNA extraction and qRT-PCR}

The total RNAs were extracted from OS cells, tissue samples or in vivo xenografts in line with the manual of TRIzol reagent (Thermo Fisher Scientific, Waltham, MA), for converting into cDNA. qRT-PCR was implemented using Power SYBR Green (TaKaRa, Shiga, Japan) on the
Step-One Plus Real-Time PCR System (Applied Biosystems, Foster City, CA). RNA expressions were calculated by $2^{-\Delta \Delta \mathrm{Ct}}$ method, and U6/GAPDH was the internal control. The primers were presented in Table 1.

\section{Transfection}

The specific shRNAs against LINC01419 (shLINC01419\#1/2) or PDRG1 (sh-PDRG1\#1/2) and pcDNA3.1-PDRG1, as well as their negative control (NC) including the nonspecific shRNA (sh-NC) and empty pcDNA3.1 vector, were all procured from RiboBio (Guangzhou, China). The miR-519a-3p mimics/inhibitor and NC mimics/inhibitor were acquired from Genepharma (Shanghai, China). Thereafter, above plasmids were transfected into the U2OS and Saos-2 cells for $48 \mathrm{~h}$ by use of Lipofectamine 2000 (Invitrogen, Carlsbad, CA), as appropriate. The sequences were shown in Table 1.

\section{Colony formation assay}

Cells were seeded in the 6-well culture plates $\left(1 \times 10^{3}\right.$ cells/well) and incubated for 2 weeks. Afterwards, colonies were colored via crystal violet solution after being fixated by $4 \%$ paraformaldehyde. Finally, colonies with over 50 cells were manually calculated.

\section{EdU assay}

Cells were plated into the 96-well culture plates and added with the EdU solution (Ribobio) for $3 \mathrm{~h}$ and then treated with $4 \%$ paraformaldehyde. Following permeabilization, cells were incubated with the DAPI solution and then analyzed by fluorescent microscope (Leica, Wetzlar, Germany).

\section{JC-1 assay}

Transfected cells were harvested for culturing with the $10 \mathrm{nM}$ of JC-1 solution (Beyotime, Shanghai, China). Following $30 \mathrm{~min}$ of incubation at $37^{\circ} \mathrm{C}$, samples were tagged with fluorescence and rinsed in PBS for EnSpire Reader analysis (PerkinElmer, Waltham, MA).

\section{TUNEL assay}

According to the instruction of in situ cell death detection kit (Roche, Basel, Switzerland), cells were fixed for $1 \mathrm{~h}$, permeabilized for $2 \mathrm{~min}$, and then cultivated with TUNEL assay buffer for $1 \mathrm{~h}$. Following DAPI staining, apoptotic cells were monitored under fluorescent microscope (Leica).

\section{Transwell assays}

Transfected cells were seeded into the top transwell inserts (Corning Incorporated, Corning, NY) with Matrigel membrane (BD Biosciences, Franklin Lakes, NJ) for invasion assay, or without Matrigel for migration 
Table 1 Sequences of primers used in qRT-PCR and of plasmids applied in cell transfection

\begin{tabular}{|c|c|}
\hline Genes & Sequences of primers $\left(5^{\prime}-3^{\prime}\right)$ \\
\hline \multirow[t]{2}{*}{ LINC01419 } & F: AGAACTGAGGTCCACTTTCTGG \\
\hline & R: GGTCCTTTGTCTGCAACGA \\
\hline \multirow[t]{2}{*}{ miR-519a-3p } & F: AAAGTGCATCCTTTTAGAGTGTGG \\
\hline & R: CTCTACAGCTATATTGCCAGCCAC \\
\hline \multirow[t]{2}{*}{$\operatorname{miR}-519 c-3 p$} & F: AAAGTGCATCTTTTTAAGAGGATCG \\
\hline & R: CTCTACAGCTATATTGCCAGCCAC \\
\hline \multirow[t]{2}{*}{ miR-519b-3p } & F: AAAGTGCATCCTTTTTAGAGGTTCC \\
\hline & R: CTCTACAGCTATATTGCCAGCCAC \\
\hline \multirow[t]{2}{*}{ miR-3121-3p } & F:TAAATAGAGTAGGCAAAGGACAGCC \\
\hline & R: CTCTACAGCTATATTGCCAGCCAC \\
\hline \multirow[t]{2}{*}{ PDRG1 } & F:TGAGCCACAAAGCACCAA \\
\hline & R:TCACATGGACCCTGAGCA \\
\hline \multirow[t]{2}{*}{ GAPDH } & F: CATCCTGGGCTACACTGAGC \\
\hline & R: AGTGGTCGTTGAGGGCAA \\
\hline \multirow[t]{2}{*}{ U6 } & F: AAAGCAAATCATCGGACGACC \\
\hline & R: GTACAACACATTGTTTCCTCGGA \\
\hline shRNAs/pcDNA3.1 & Sequences $\left(5^{\prime}-3^{\prime}\right)$ \\
\hline sh-NC & CCGGCACTAATGTCTGCGTCAATGTCTCGAGACATTGACGCAGACATTAGTGTTTTTTG \\
\hline sh-LINC01419\#1 & CCGGTGCATAGATTCATTAAGAATTCTCGAGAATTCTTAATGAATCTATGCATTTTTG \\
\hline sh-LINC01419\#2 & CCGGATCTATGAAGCCAAACCTAATCTCGAGATTAGGTTTGGCTTCATAGATTTTTTG \\
\hline sh-NC & CCGGGTGACATAGTGCACAGTTGGACTCGAGTCCAACTGTGCACTATGTCACTTTTTG \\
\hline sh-PDRG1\#1 & CCGGCACCAAGATTCTGTTCTTCATCTCGAGATGAAGAACAGAATCTTGGTGTTTTTG \\
\hline sh-PDRG1\#2 & CCGGACCAAGATTCTGTTCTTCATTCTCGAGAATGAAGAACAGAATCTTGGTTTTTTG \\
\hline pcDNA3.1-P DRG1 & $\begin{array}{l}\text { ATGCTATCACCCGAGGCAGAGCGAGTGCTGCGGTACCTTGTAGAAGTGGAGGAG } \\
\text { CTCGCCGAGGAGGTGCTGGCGGACAAGCGGCAGATTGTGGACCTGGACACTAAA } \\
\text { AGGAATCAGAATCGAGAGGGCCTGAGGGCCCTGCAGAAGGATCTCAGCCTCTCT } \\
\text { GAAGATGTGATGGTTTGCTTCGGGAACATGTTTATCAAGATGCCTCACCCTGAG } \\
\text { ACAAAGGAAATGATTGAAAAAGATCAAGATCATCTGGATAAAGAAATAGAAAAA } \\
\text { CTGCGGAAGCAACTTAAAGTGAAGGTCAACCGCCTTTTGAGGCCCAAGGCAAA } \\
\text { CCGGAGCTGAAGGGTTTTAACTTGAACCCCCTCAACCAGGATGAGCTTAAAGCT } \\
\text { CTCAAGGTCATCTTGAAAGGA }\end{array}$ \\
\hline \multicolumn{2}{|l|}{ Mimics/inhibitor } \\
\hline NC mimics & AUUCACGUUCCUUUUAGAGUGU \\
\hline miR-519a-3p mimics & AAAGUGCAUCCUUUUAGAGUGU \\
\hline NC inhibitor & ACACUCUAAAAGGAACGUGAAU \\
\hline miR-519a-3p inhibitor & ACACUCUAAAAGGAUGCACUUU \\
\hline
\end{tabular}

assay. The complete medium was supplemented to the bottom inserts. $24 \mathrm{~h}$ post-incubation, migrated or invaded cells were treated with $4 \%$ paraformaldehyde and crystal violet for observation under microscope.

\section{Immunofluorescence (IF)}

Cells were seeded in the 12-well culture plates, and then processed with $4 \%$ formaldehyde in PBS, with $0.5 \%$ Triton $\mathrm{X}-100$ for $10 \mathrm{~min}$. Following being mixed with $5 \%$ bovine serum albumin (BSA) for $30 \mathrm{~min}$, the specific antibodies to E-cadherin (ab76055, 1:500 dilution; Abcam, Cambridge, UK) and N-cadherin (ab76057, 1:500 dilution; Abcam), as well as the secondary antibodies (ab205719,
1:2000 dilution; Abcam) were used in sequence. Samples treated with DAPI solution were analyzed under microscope.

\section{Western blot}

Total proteins were extracted from U2OS and Saos-2 cells by RIPA buffer. Then, extracted proteins were separated using SDS-PAGE and transferred onto PVDF membranes (Millipore, Billerica, MA, USA). After being sealed via $5 \%$ skim milk, the membranes were processed with primary antibodies (Abcam, Cambridge, USA) overnight at $4{ }^{\circ} \mathrm{C}$. Later, the membranes were added with secondary antibodies (ab205719, 1:10000; Abcam) at $37{ }^{\circ} \mathrm{C}$ 
in a dark room for $1 \mathrm{~h}$ of incubation. At last, the protein bands were visualized by enhanced ECL system (Tanon, Shanghai, China). The internal control was GAPDH. Primary antibodies were as below: E-cadherin (ab76055, 1:1000 dilution), N-cadherin (ab76057, 1:1000 dilution), Vimentin (ab92547, 1:2000 dilution), $\beta$-catenin (ab16051, 1:2000 dilution), GAPDH (ab181602, 1:10000 dilution). All these antibodies were all purchased from Abcam.

\section{In vivo assay}

Ten BALA/C nude male mice (aged 4-6 weeks; weighted 18-24 g) were acquired from Shi Laike Company (Shanghi, China). Then, the mice were randomly divided into 2 groups ( $\mathrm{n}=5$ per group) and injected subcutaneously with U2OS cells which were stably transfected with sh-LINC01419 or sh-NC. Every 4 days, tumor size was monitored and recorded. 28 days later, the mice were sacrificed through cervical dislocation and the tumor was excised and weighed. Tumor volume was assessed according to the formula $\left(\mathrm{mm}^{3}\right)$ : tumor volume $=$ length $\times$ width $^{2} \times 0.5$. The animal experiments were approved by Hwa Mei Hospital, University of Chinese Academy of Sciences (Ningbo NO.2 Hospital).

\section{Immunohistochemistry (IHC)}

IHC was conducted as described previously [20]. Briefly, sections from paraffin-embedded xenografts were processed with primary antibodies against Ki-67 (ab15580, 1:500 dilution; Abcam) and PCNA (ab92552, 1:500 dilution; Abcam). The proteins in situ were observed with the application of Super Sensitive Link-Label IHC Detection System (BioGenex, Fremont, CA, USA).

\section{FISH}

The FISH probe designed for LINC01419 was commercially obtained from Ribobio and used as per the user guide. DAPI solution was added for nuclear counterstaining. Images were captured under microscope.

\section{Subcellular fractionation}

Based on the supplier's instruction, PARIS ${ }^{\mathrm{TM}}$ Kit (Invitrogen) was employed for subcellular fractionation assay. The distribution of RNAs in nuclei and cytoplasm was monitored by qRT-PCR.

\section{MS2-RIP assay}

Cells were transfected, and then harvested for the cotransfection with pcDNA3.1-MS2 and pcDNA3.1-MS2LINC01419 for $48 \mathrm{~h}$. Samples were then subjected to RIP assay with GFP antibody (ab290, 1:200 dilution; Abcam) by use of RIP RNA-Binding Protein Immunoprecipitation
Kit (Millipore, Bedford, MA). Finally, qRT-PCR was exploited to analyze the level of co-precipitated RNAs.

\section{RNA pull down assay}

The wild-type (WT) and mutated (Mut) miR-519a-3p fragments covering the LINC01419 or PDRG1 were synthesized and biotinylated to Bio-miR-519a-3p-WT/Mut. The probes were cultivated with the protein extracts from U2OS and Saos- 2 cells, followed by incubation with the streptavidin agarose magnetic beads for $1 \mathrm{~h}$. RNA enrichment in pull-downs was detected via qRT-PCR.

\section{Dual-luciferase reporter gene analyses}

The recombinant luciferase reporter vectors LINC01419WT/Mut and PDRG1-WT/Mut were severally established by using the wild-type and mutated LINC01419 or PDRG1 fragments covering miR-519a-3p binding sites. The Dual-Luciferase miRNA Target Expression Vector pmirGLO was obtained from Promega (Madison, WI). The reporter vectors were co-transfected with miR-519a-3p mimics or NC mimics for $48 \mathrm{~h}$, and then luciferase activity of the reporters was monitored via Dual-Luciferase Reporter Assay System (Promega), respectively.

\section{Statistical analyses}

Data from three independent bio-triplications were exhibited as the mean \pm standard deviation (SD). Statistical difference was analyzed by Student's $t$ test or one-way ANOVA via PRISM 6 (GraphPad, San Diego, CA), with the $\mathrm{p}<0.05$ meant statistically significant.

\section{Results}

LINC01419 acts as an oncogene in OS

Firstly, we determined the expression pattern of LINC01419 in OS. It was showed that LINC01419 exhibited a higher expression level in OS tissues than in adjacent non-tumor tissues (Additional file 1: Figure S1A). Then, we checked LINC01419 expression in OS cell lines. As a result, LINC01419 was significantly up-regulated in OS cell lines relative to normal hFOB1.19 cells, especially in U2OS and Saos-2 cells (Fig. 1a). Based on above results, we wanted to further explore the biological role of LINC01419 in OS. Prior to that, we confirmed that LINC01419 was successfully silenced in U2OS and Saos- 2 cells after transfection with sh-LINC01419\#1/2 (Fig. 1b). Then, the proliferation of U2OS and Saos-2 cells was determined by colony formation assay and EdU assay. The results manifested that LINC01419 suppression led to inhibited OS cell proliferation (Fig. 1c, d). By contrast, JC-1 and TUNEL assays revealed that the apoptosis of U2OS and Saos-2 cells was hastened upon 


\section{(See figure on next page.)}

Fig. 1 LINC01419 acts as an oncogene in OS. a LINC01419 expression was assessed in OS cell lines (143B, U2OS and Saos-2) and human normal osteoblast (hFOB1.19) by using qRT-PCR analysis. b Silencing efficiency of LINC01419 was evaluated in U2OS and Saos-2 cells by qRT-PCR. c-d. Colony formation assay and EdU assay (scale bar $=200 \mu \mathrm{m}$ ) were conducted to evaluate the proliferation of U2OS and Saos-2 cells transfected with sh-LINC01419\#1/2 or sh-NC. e-f. U2OS and Saos-2 cell apoptosis was examined by JC-1 assay (scale bar = $200 \mu$ m) and TUNEL assay (scale bar $=200 \mu \mathrm{m}$ ) in sh-LINC01419 group or sh-NC group. g-h. U2OS and Saos-2 cells migration and invasion were illustrated by transwell assay (scale bar $=200 \mu \mathrm{m}$ ) with the transfection sh-LINC01419 or sh-NC. i-j. IF (scale bar $=50 \mu \mathrm{m})$ and western blot assay were conducted to investigate the EMT process of sh-LINC01419 or sh-NC transfected U2OS and Saos-2 cells. ${ }^{*} P<0.05,{ }^{*} P<0.01$

LINC01419 knockdown (Fig. 1e, f). Moreover, transwell assay delineated that the migration and invasion were curbed in U2OS and Saos-2 cells after silencing LINC01419 (Fig. 1g, h). Through IF assay, we observed that EMT process was hampered by silenced LINC01419 (Fig. 1i). Furthermore, western blot analysis indicated that LINC01419 deficiency augmented the protein level of E-cadherin but lowered the protein levels of N-cadherin, Vimentin and $\beta$-catenin (Fig. 1j). These results suggested that LINC01419 enhanced OS cell malignancies in vitro. Meanwhile, we carried out in vivo assay to further validate the contributing role of LINC01419 in OS. Consequently, tumors excised from mice injected with LINC01419-silenced cells were much smaller than those from control group (Additional file 1: Figure S1B). Also, decreased volume and weight was observed in tumors from group with LINC01419 depletion compared to controls (Additional file 1: Figure S1C-D). In addition, it was confirmed that LINC01419 expression in tumors originated from sh-LINC01419\#1-transfected cells was much less than in those from control group (Additional file 1: Figure S1E). Moreover, IHC assay implied that LINC01419 silencing reduced the positivity of PCNA and Ki-67 in in vivo tumors (Additional file 1: Figure S1F). Taken all together, LINC01419 served as an oncogene in OS.

\section{LINC01419 sponges miR-519a-3p in OS}

Next, we explored the molecular mechanism of LINC01419 in OS. It has been evidenced that IncRNA could serve as a ceRNA to up-regulate mRNA expression via sponging miRNAs in the cytoplasm [21,22]. First of all, we evaluated the distribution of LINC01419 by FISH and subcellular fractionation analyses. The results disclosed that a larger proportion of LINC01419 was located in the cytoplasm than in nucleus of both the two OS cells (Fig. 2a, b). Thence, we speculated that LINC01419 might act as a miRNA sponge. According to ENCORI database (http://starbase.sysu.edu.cn/index.php), four potential miRNAs (miR-519a-3p, miR-519c-3p, miR-519b-3p and miR-3121-3p) were postulated to bind to LINC01419 (Fig. 2c). To screen out the miRNAs interacted with LINC01419 in OS, MS2-RIP assay was conducted and verified the abundant enrichment of miR-519a-3p and
miR-519c-3p in LINC01419-precipitated compounds in these two cells (Fig. 2d). Through qRT-PCR assay, miR$519 a-3 p$ was found to be lowly expressed in OS cells while miR-519c-3p showed no expression difference (Fig. 2e). Therefore, miR-519a-3p was chosen for further exploration. As demonstrated in Fig. 2f, binding sites between LINC01419 and miR-519a-3p were predicted via ENCORI database. RNA pull down assay uncovered a direct interaction between miR-519a-3p and LINC01419 whereas mutated miR-519a-3p had no capacity to bind with LINC01419 (Fig. 2g). Moreover, miR-519a-3p overexpression efficiency was validated by qRT-PCR assay (Fig. 2h). Luciferase reporter assay verified that luciferase activity of LINC01419-WT was significantly lessened by ectopic expression of miR-519a-3p whereas no difference was presented in that of LINC01419-Mut (Fig. 2i). In conclusion, LINC01419 could sponge miR-519a-3p in OS.

\section{PDRG1 is targeted by miR-519a-3p and promotes OS cell progression}

We further investigated the target genes of miR-519a-3p in OS. Combining with four online tools (PITA, miRmap, microT and RNA22), 16 mRNAs were predicted as the targets of miR-519a-3p (Fig. 3a). Subsequently, among the 16 mRNAs, PDRG1 was screened out since it was the only one that could be regulated by both LINC01419 and miR-519a-3p (Fig. 3b). Hence, we hypothesized that PDRG1 was the downstream molecule of LINC01419/ miR-519a-3p axis in OS. Later, we found that PDRG1 was highly expressed in OS cells (Fig. 3c). Furthermore, the binding sequences of miR-519a-3p on PDRG1 $3^{\prime}$ UTR was presented in Fig. 3d. Results of RNA pull down assay indicated that PDRG1 was enriched in Bio-miR-519a$3 p-W T$ group rather than Bio-miR-519a-3p-Mut group (Fig. 3e). Luciferase reporter assay further confirmed that upregulation of miR-519a-3p could lessen the luciferase activity of PDRG1-WT but not that of PDRG1-Mut (Fig. 3f). Then, we knocked down PDRG1 expression in OS cells to probe the function of PDRG1 in OS (Fig. 3G). It was discovered that OS cell proliferation was overtly repressed by PDRG1 deficiency (Fig. 3h, i). Besides, the apoptosis of OS cells was accelerated in response to PDRG1 inhibition (Fig. 3j, k). Moreover, we found that 


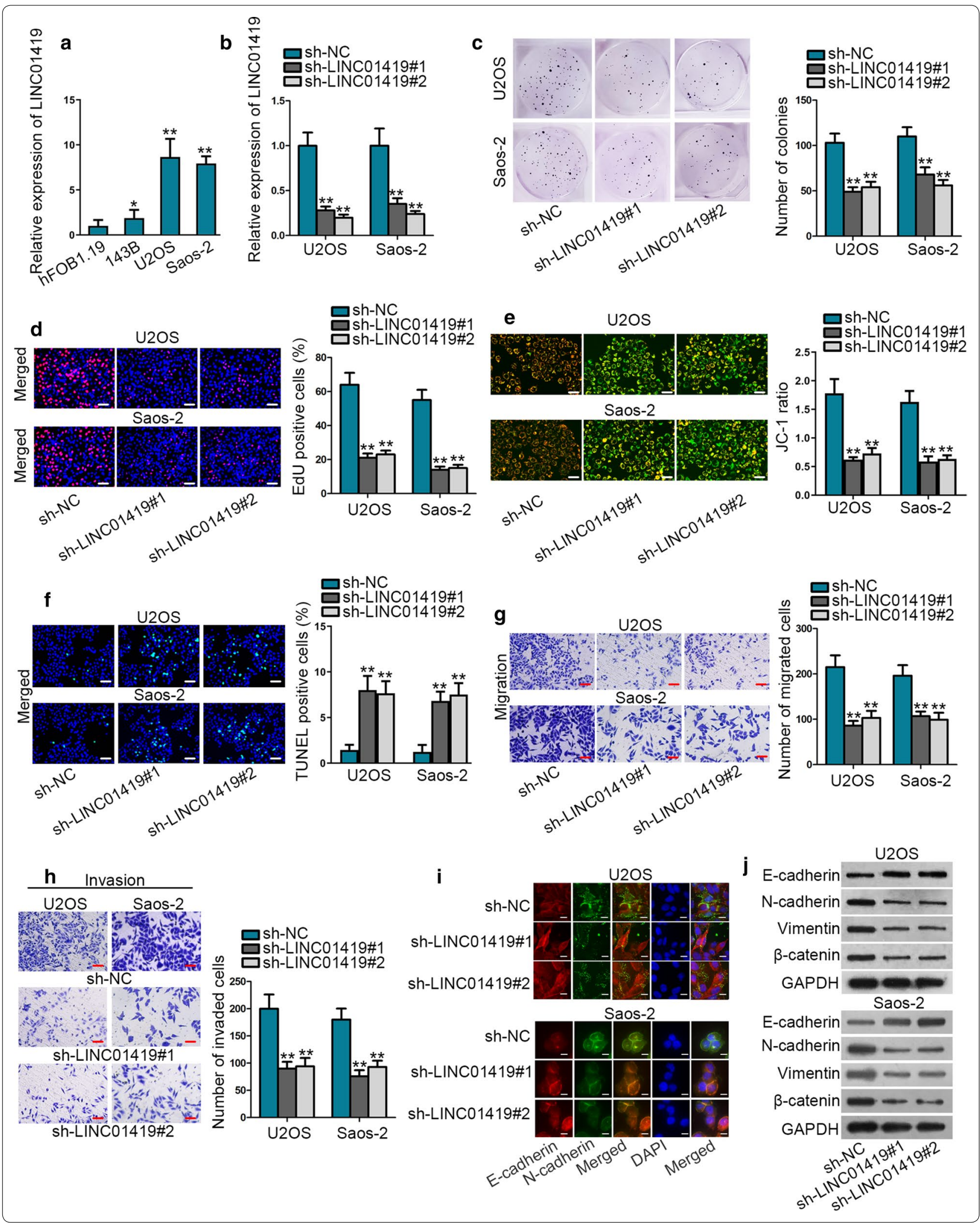



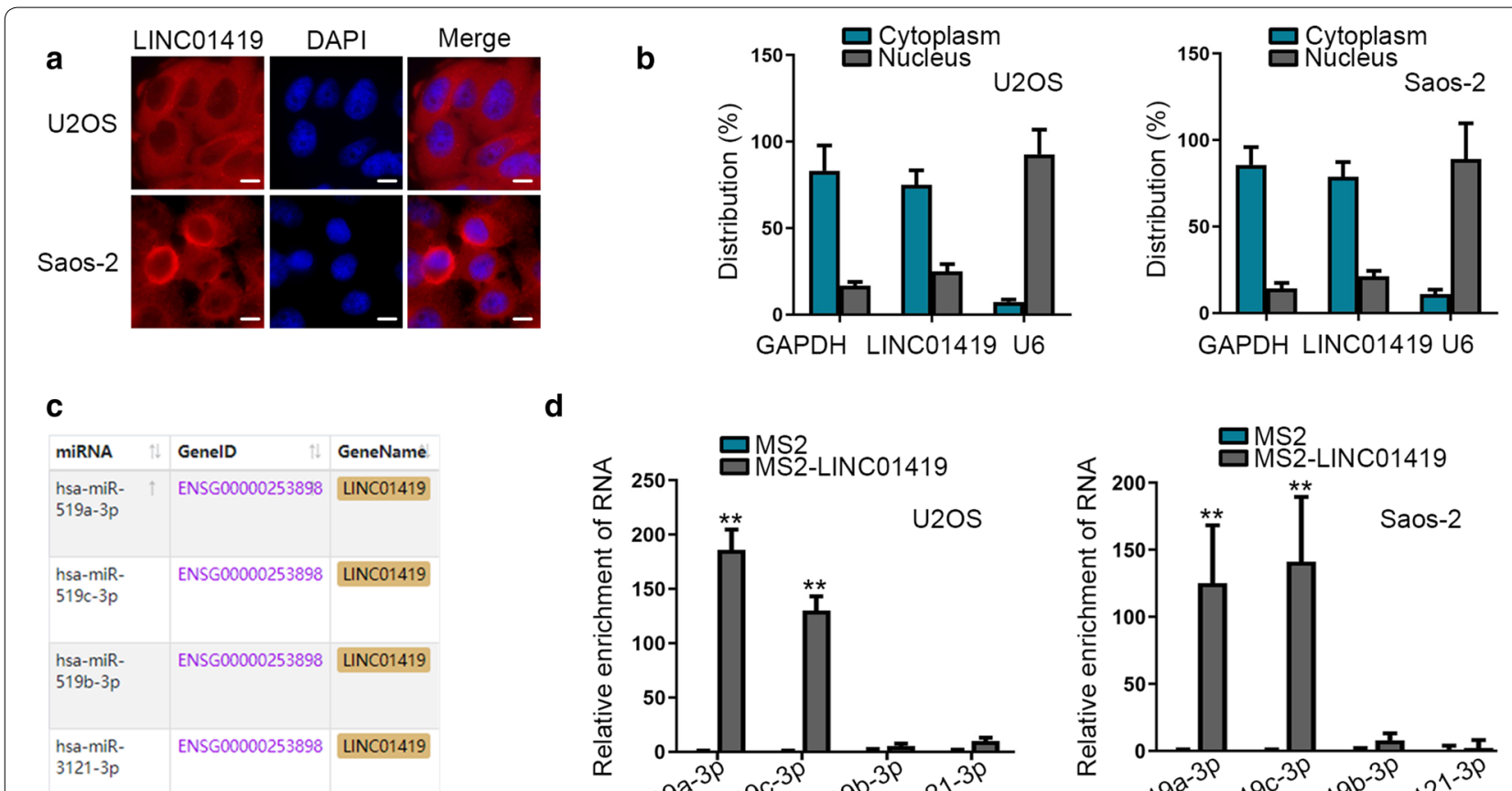

d
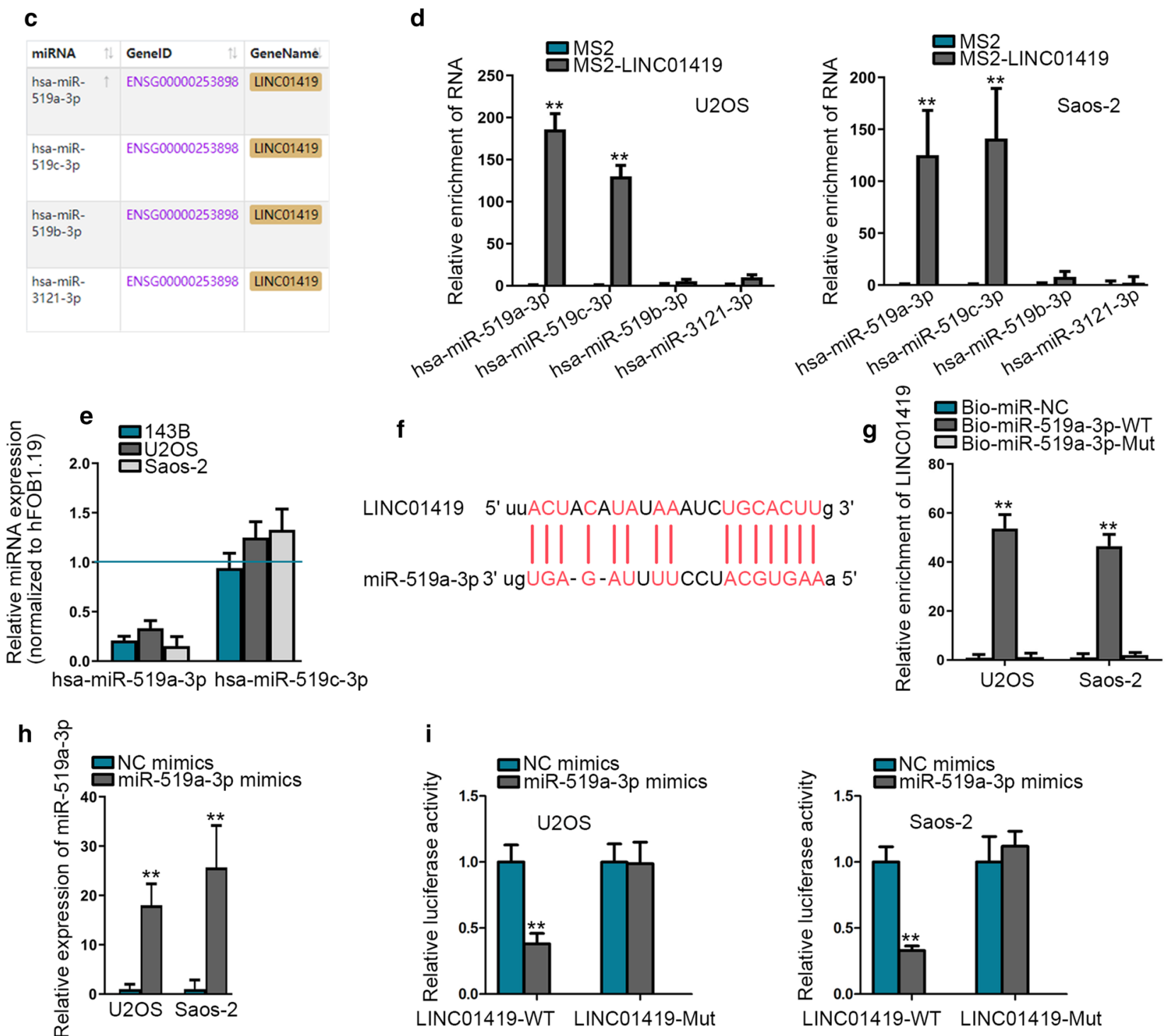

Fig. 2 LINC01419 sponges miR-519a-3p in OS. a-b FISH assay (scale bar $=50 \mu \mathrm{m}$ ) and subcellular fractionation assay were implemented to determine the location of LINC01419 in OS cells. $\mathbf{c}$ Four miRNAs that bound to LINC01419 were screened out from ENCORI database. d MS2-RIP assay was conducted to assess the binding of 4 mirnas to linc01419. e Expressions of miR-519a-3p and miR-519c-3p in OS cells were investigated via qRT-PCR. $\mathbf{f}$ Binding sites between LINC01419 and miR-519a-3p were predicted by ENCORI database. $\mathbf{g}$ RNA pull down assay was implemented to evaluate the interaction between LINC01419 and miR-519a-3p in OS cells. $\mathbf{h}$ Overexpression efficiency of miR-519a-3p in U2OS and Saos-2 cells was examined by qRT-PCR. $\mathbf{i}$ Luciferase reporter assay verified the binding of LINC01419 to miR-519a-3p in U2OS and Saos-2 cells. ${ }^{* *} P<0.01$ 
(See figure on next page.)

Fig. 3 PDRG1 is targeted by miR-519a-3p and promotes OS cell progression. a Venn diagram was generated to show the potential mRNAs targeted by miR-519a-3p. b The expressions of mRNAs regulated by LINC01419 or miR-519a-3p were shown by qRT-PCR. c qRT-PCR analysis was conducted to estimate the expression of PDRG1 in OS cells. $\mathbf{d}$ Binding site between PDRG1 and miR-519a-3p was presented. e-f. RNA pull down assay and luciferase reporter assay assessed the combination of PDRG1 with miR-519a-3p. $\mathbf{g}$ Inhibition efficiency of PDRG1 was detected by qRT-PCR. $\mathbf{h}-\mathbf{i}$. Effect of PDRG1 silencing on U2OS and Saos-2 cell proliferation was evaluated by colony formation and EdU assays. $\mathbf{j}-\mathbf{k}$. The apoptosis of U2OS and Saos-2 cells was examined by JC-1 and TUNEL assays with or without PDRG1 silence. I- $\mathbf{m}$. The migration and invasion in indicated cells were tested by transwell assay. $\mathbf{n}$. IF assay (scale bar $=50 \mu \mathrm{m}$ ) was performed to investigate EMT process in U2OS and Saos-2 cells upon PDRG1 knockdown. ${ }^{* *} P<0.01$

silencing PDRG1 restrained the motility of OS cells (Fig. 3l, m). Additionally, EMT process was inhibited in OS cells by PDRG1 interfering (Fig. 3n). To be concluded, PDRG1 was targeted by LINC01419/miR-519a-3p signaling and played an oncogenic role in OS.

\section{LINC01419 facilitates OS development through targeting miR-519a-3p/PDRG1 axis}

Finally, we tested the function of LINC01419/miR519a-3p/PDRG1 axis in OS. At first, we discovered that PDRG1 expression was reduced when LINC01419 was inhibited or miR-519a-3p was overexpressed (Fig. 4a), which confirmed LINC01419/miR-519a-3p/PDRG1 axis in OS. Therefore, restoration experiments were designed and carried out. Prior to that, inhibition efficiency of miR-519a-3p and overexpression efficiency of PDRG1 were validated via qRT-PCR assay (Fig. 4b, c). Based on the results from colony formation and EdU assays, the decreased proliferation caused by LINC01419 inhibition was countervailed by inhibited miR-519a-3p or overexpressed PDRG1 (Fig. 4d, e). On the contrary, JC-1 and TUNEL assays displayed that LINC01419 suppressionaugmented apoptosis was offset by miR-519a-3p inhibition or PDRG1 upregulation (Fig. 4f, g). Moreover, miR-519a-3p inhibition or PDRG1 overexpression rescued the restrained motility of LINC01419-depleted OS cells (Fig. 4h, i). Lastly, IF and western blot assays proved that suppressed miR-519a-3p or overexpressed PDRG1 abolished the inhibitory effect of LINC01419 depletion on EMT process (Fig. 4j, k). In conclusion, LINC01419/ miR-519a-3p/PDRG1 axis exacerbated cell development in OS.

\section{Discussion}

OS is a musculoskeletal tumor originating in the interstitial cell with a high risk of death [23]. Emerging studies indicated that lncRNAs are closely associated with biological progression of various cancers [24]. It has been reported that lncRNAs with aberrant expression can serve as tumor promoter or suppressor in multiple cancers, including OS $[25,26]$. As an example, lncRNA FER1L4 was under-expressed in OS and repressed OS cell proliferation, migration and invasion by miR-18a-5p/
PTEN axis [27]. LncRNA B4GALT1-AS1 was up-regulated and contributed to cell migration and stemness in OS by stabilizing YAP mRNA [28]. Similarly, here we found that LINC01419 level was remarkably enhanced in OS tissues and cells. Besides, knockdown of LINC01419 impaired OS cell proliferation, migration, invasion and EMT process while boosted cell apoptosis. Furthermore, LINC01419 silencing inhibited OS tumor growth. These results revealed the oncogenic property of LINC01419 in OS, consistent with its role reported in lung adenocarcinoma, esophageal squamous cell carcinoma and gastric cancer [17-19].

MiRNAs are identified as another subtype of ncRNAs with 20-24 nucleotides in length and play crucial roles in cancer malignant process [29, 30]. Extensive evidence has proved that lncRNAs could serve as a ceRNA of certain miRNAs to block the silence of miRNA downstream genes [31, 32]. The regulatory mechanism between miRNAs and lncRNAs has been widely reported in OS. As illustrated, lncRNA FBXL19-AS1 facilitated cell proliferation and invasion in OS through targeting miR-346 [33]. LncRNA NEAT1 hastened EMT process and predicted poor prognosis by sponging miR-186-5p in OS [34]. The present study indicated that LINC01419 was a cytoplasmic lncRNA that could interact with miR-519a-3p in OS. Importantly, miR-519a-3p was proved to possess a low expression in OS cells. Above data unveiled that LINC01419 exerted a sponging function in OS.

P53 and DNA damage regulated 1 (PDRG1) has been uncovered to accelerate the progression of lung cancer [35]. In addition, PDRG1 functioned as an proliferationfacilitator in gastric cancer [36]. This study uncovered the high level and tumor-facilitating role of PDRG1 in OS cells. Besides, we recognized PDRG1 as the target downstream of LINC01419/miR-519a-3p axis in OS. Restoration assays further confirmed that LINC01419 contributed to OS cell progression via miR-519a-3p/ PDRG1 pathway.

\section{Conclusion}

In conclusion, our research was the first to validate the biological function and molecular mechanism of LINC01419 in OS, and confirmed the carcinogenic 


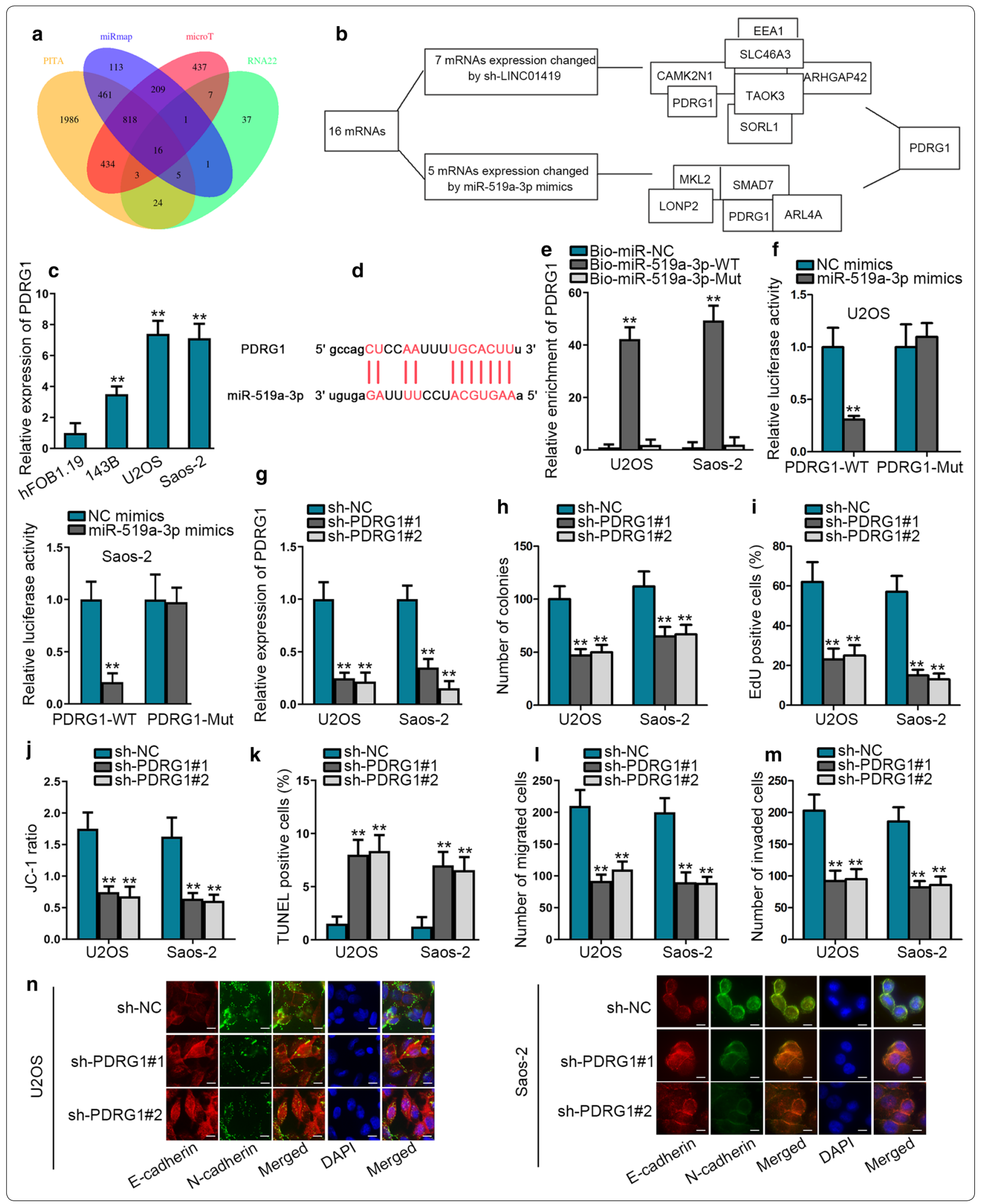



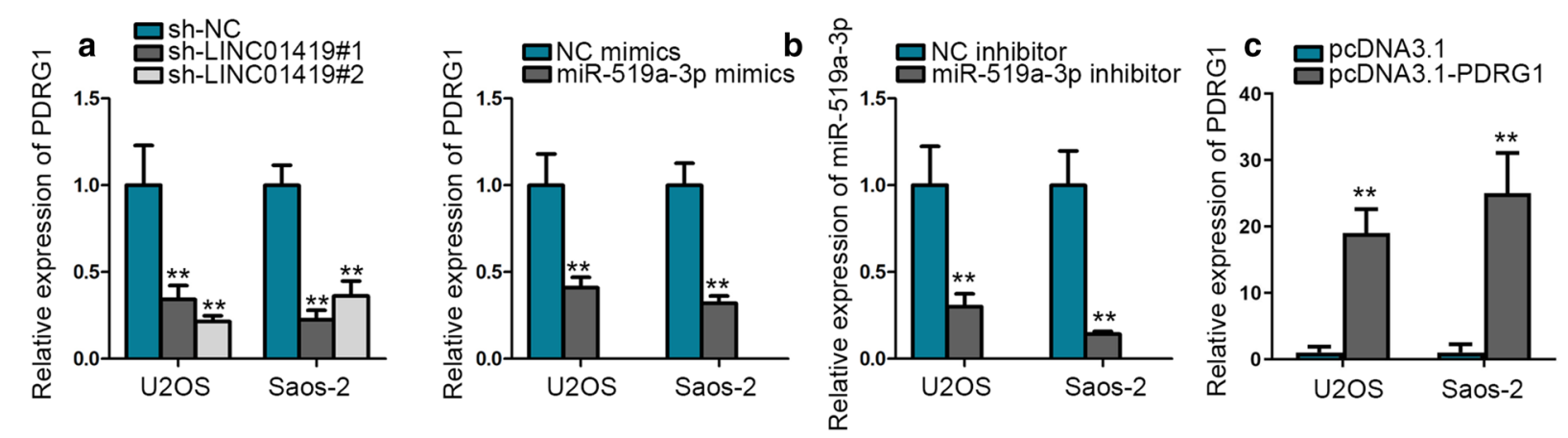

d

e

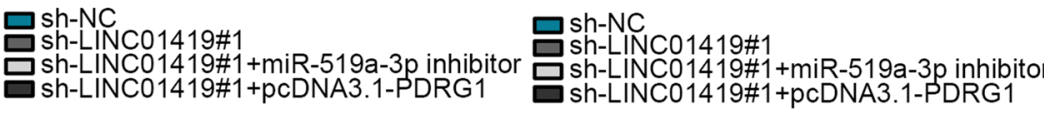

马 sh-LINC01419\#1 +miR-519a-3p inhibitor

号 sh-LINC01419\#1+pcDNA3.1-PDRG1
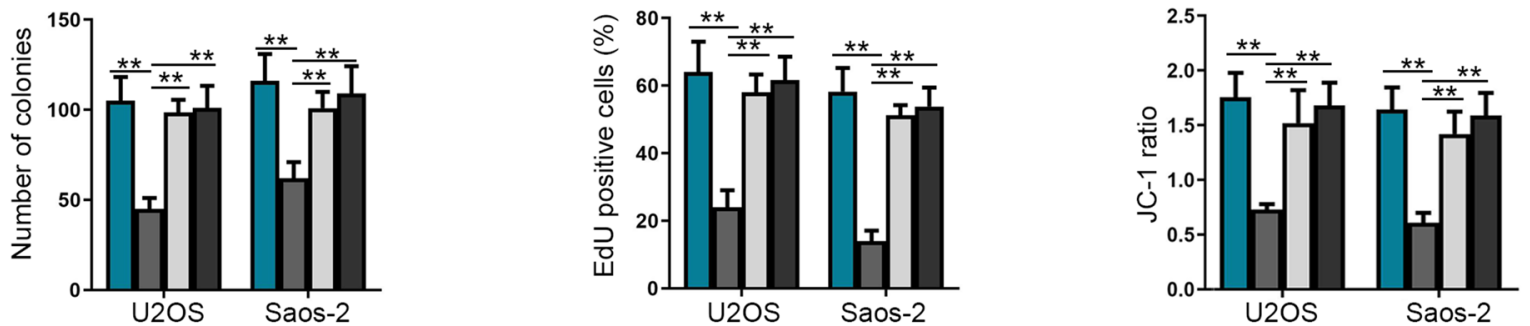

g

h
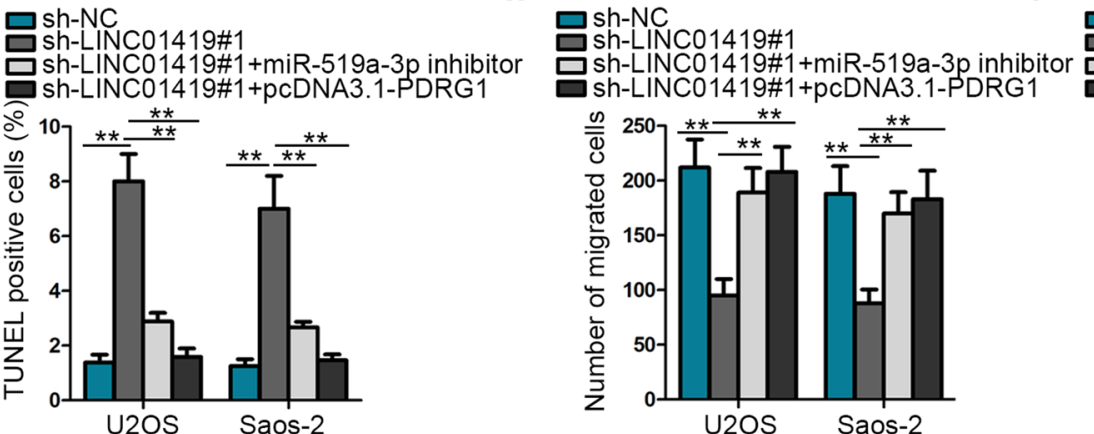

Sh-LINC01419\#1

sh-LiNC01419\#1+miR-519a-3p inhibitol sh-LINC01419\#1+pcDNA3.1-PDRG1 sh-LINC01419\#1+pcDNA3.1-PDRG1
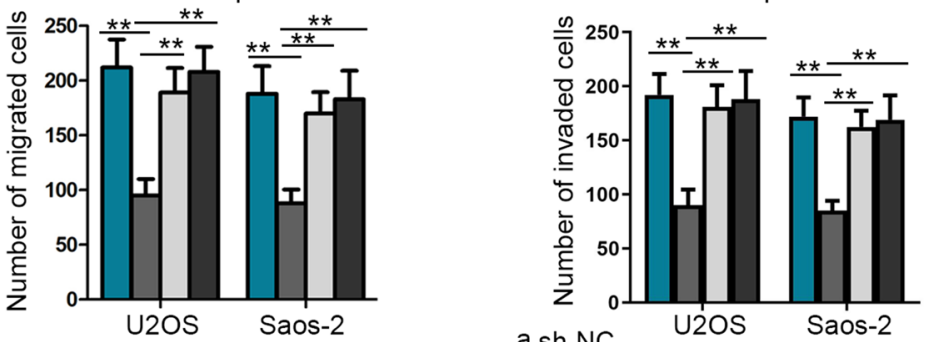

sh-LINC01419\#1

sh-LINC01419\#1+miR-519a-3p inhibito
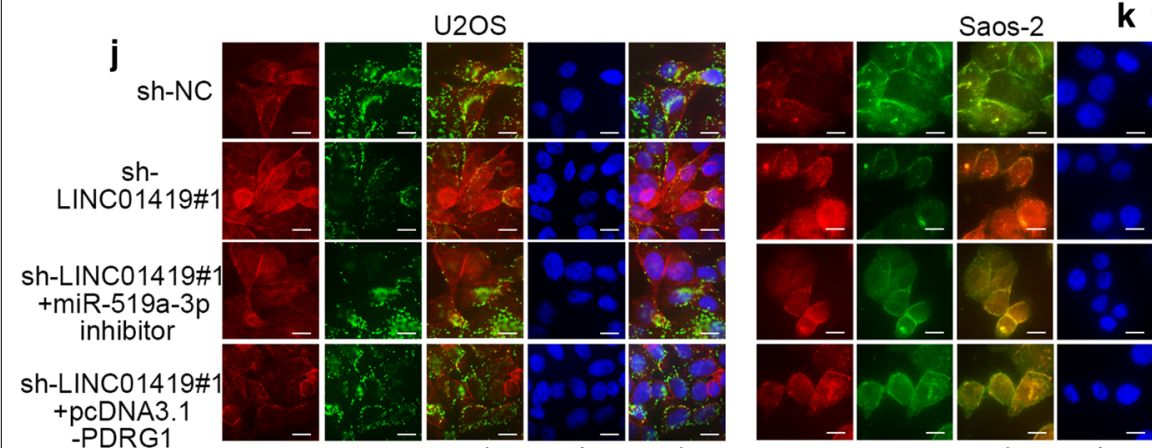

d sh-LINC01419\#1+pcDNA3.1-PDRG1
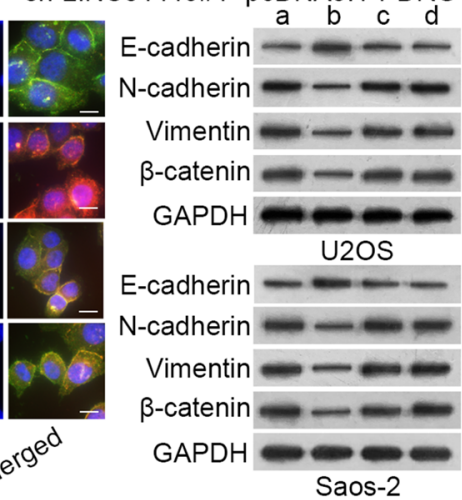

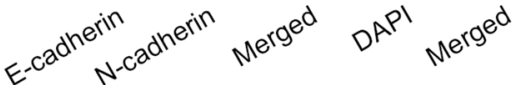

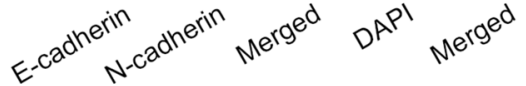

Saos-2

Fig. 4 LINC01419 contributes to malignant phenotypes of OS cells through targeting miR-519a-3p/PDRG1 axis. a Effect of LINC01419 depletion or miR-519a-3p upregulation on PDRG1 expression was estimated by qRT-PCR. $\mathbf{b}-\mathbf{c}$ qRT-PCR analysis was used to determine the transfection efficiency of miR-519a-3p inhibitor or pCDNA3.1-PDRG1. d-e. Cell proliferation in each group was evaluated by colony formation and EdU assays. $\mathbf{f}-\mathbf{g}$. JC-1 and TUNEL assays were carried out to assess cell apoptosis in each group. $\mathbf{h}-\mathbf{i}$. The motility of cells under indicated transfections was detected by transwell assay. j-k. IF assay (scale bar $=50 \mu \mathrm{m}$ ) and western blot analysis were employed to test changes on EMT process in each group. ${ }^{* *} P<0.01$ 
function of LINC01419/miR-519a-3p/PDRG1 axis in OS development. This finding might provide a theoretic basis for the investigation of OS treatment. However, our study was lack of the exploration on the upstream mechanism of LINC01419 which we would explore in the future.

\section{Supplementary information}

Supplementary information accompanies this paper at https://doi. org/10.1186/s12935-020-01203-0.

Additional file 1: Figure S1. A. LINC01419 expression in 50 pairs of OS tissues and adjacent non-tumor tissues. B. Representative images of tumors excised from sh-LINC01419 and sh-NC group. C-D. Tumor volume and weight were measured in sh-LINC01419 and sh-NC group. E. LINC01419 expression was detected in tumors from sh-LINC01419 and sh-NC group. F. The levels of Ki-67 and PCNA were determined by IHC assay (scale bar $=100 \mu \mathrm{m}){ }^{* *} P<0.01$.

\section{Abbreviations}

OS: Osteosarcoma; IncRNAs: Long noncoding RNAs; LINC01419: Long intergenic non-protein coding RNA 1419; EMT: Epithelial-mesenchymal transition; qRT-PCR: Quantitative real-time polymerase chain reaction; IF: Immunofluorescence; ceRNA: Competing endogenous RNA; miRNAs: MicroRNAs; DMEM: Dulbecco's Modified Eagle's Medium; FBS: Fetal bovine serum; GAPDH: Glyceraldehyde-3-phosphate dehydrogenase; shRNA: Short hairpin RNA; EdU: 5-ethynyl-2'-deoxyuridine; PBS: Phosphate buffer saline; SDS-PAGE: Sodium dodecyl sulfate-polyacrylamide gel electrophoresis; PVDF: Polyvinylidene fluoride; IHC: Immunohistochemistry; FISH: Fluorescent in situ hybridization; WT: Wild type; Mut: Mutant type; SD: Standard deviation; ANOVA: One-way analysis of variance; PDRG1: p53 and DNA damage regulated 1.

\section{Acknowledgements}

We sincerely appreciate all individuals and groups involved in this study.

\section{Authors' contributions}

ZG: study design, data curation, interpretation, manuscript review; SW, JW and SZ: experiments, data record and analysis, draft, figures. All authors have made substantial contributions to this study. All authors read and approved the final manuscript.

\section{Funding}

This study was supported by Reasearch Foundation of Hwa Mei Hospital, University of Chinese Academy of Sciences, China (Grant Nos. 2020HMKY44, 2020HMKY06); Natural Science Foundation of Zhejiang, China (Grant No. LQ20H060002); and Ningbo Institute of Life and Health Industry, University of Chinese Academy of Sciences.

\section{Ethics approval and consent to participate}

This research was approved by the Ethics committee of Hwa Mei Hospital, University of Chinese Academy of Sciences (Ningbo NO.2 Hospital). All patients involved in our study have signed the informed consents.

\section{Consent for publication}

All authors have agreed the publication of this manuscript.

\section{Competing interests}

Authors state no conflicts of interest in this study.

\section{Author details}

${ }^{1}$ Department of Orthopedics, Hwa Mei Hospital, University of Chinese Academy of Sciences (Ningbo NO. 2 Hospital), No. 41 Northwest Street, Ningbo 315010, Zhejiang, China. ${ }^{2}$ Ningbo Institute of Life and Health Industry, No. 41 Northwest Street, Ningbo 315010, Zhejiang, China.

\section{References}

1. Siegel RL, Miller KD, Jemal A. Cancer statistics. CA Cancer J Clin. 2015;65(1):5-29.

2. Cho Y, Jung GH, Chung SH, Kim JY, Choi Y, Kim JD. Long-term survivals of stage Ilb osteosarcoma: a 20-year experience in a single institution. Clin Orthop Surg. 2011;3(1):48-54.

3. Savage SA, Mirabello L. Using epidemiology and genomics to understand osteosarcoma etiology. Sarcoma. 2011;2011:548151.

4. Broadhead ML, Clark JC, Myers DE, Dass CR, Choong PF. The molecular pathogenesis of osteosarcoma: a review. Sarcoma. 2011;2011:959248.

5. Djebali S, Davis CA, Merkel A, Dobin A, Lassmann T, Mortazavi A, Tanzer A, Lagarde J, Lin W, Schlesinger F, et al. Landscape of transcription in human cells. Nature. 2012;489(7414):101-8.

6. Esteller M. Non-coding RNAs in human disease. Nat Rev Genet. 2011;12(12):861-74.

7. Anastasiadou E, Jacob LS, Slack FJ. Non-coding RNA networks in cancer. Nat Rev Cancer. 2018;18(1):5-18.

8. Iorio MV, Croce CM. Causes and consequences of microRNA dysregulation. Cancer J (Sudbury, Mass). 2012;18(3):215-22.

9. Harries LW. Long non-coding RNAs and human disease. Biochem Soc Trans. 2012;40(4):902-6.

10. Lei H, Gao Y, Xu X. LncRNA TUG1 influences papillary thyroid cancer cell proliferation, migration and EMT formation through targeting miR-145. Acta Biochim Biophys Sin. 2017;49(7):588-97.

11. Jin Y, Feng SJ, Qiu S, Shao N, Zheng JH. LncRNA MALAT1 promotes proliferation and metastasis in epithelial ovarian cancer via the PI3K-AKT pathway. Eur Rev Med Pharmacol Sci. 2017;21(14):3176-84.

12. Zhu H, Zhao H, Zhang L, Xu J, Zhu C, Zhao H, Lv G. Dandelion root extract suppressed gastric cancer cells proliferation and migration through targeting IncRNA-CCAT1. Biomed Pharmacother. 2017;93:1010-7.

13. Sand M, Bechara FG, Sand D, Gambichler T, Hahn SA, Bromba M, Stockfleth E, Hessam S. Long-noncoding RNAs in basal cell carcinoma. Tumour Biol. 2016;37(8):10595-608.

14. Lemos AE, Ferreira LB, Batoreu NM, de Freitas PP, Bonamino MH, Gimba ER. PCA3 long noncoding RNA modulates the expression of key cancer-related genes in LNCaP prostate cancer cells. Tumour Biol. 2016;37(8):11339-48.

15. Wang D, Hu Y. Long non-coding RNA PVT1 competitively binds MicroRNA-424-5p to regulate CARM1 in radiosensitivity of non-small-cell lung cancer. Mol Ther Nucleic Acids. 2019;16:130-40.

16. Gao J, Zeng K, Liu Y, Gao L, Liu L. LncRNA SNHG5 promotes growth and invasion in melanoma by regulating the miR-26a-5p/TRPC3 pathway. OncoTargets Ther. 2019;12:169-79.

17. Wang LL, Zhang L, Cui XF. Downregulation of long noncoding RNA LINC01419 inhibits cell migration, invasion, and tumor growth and promotes autophagy via inactivation of the PI3K/Akt1/mTOR pathway in gastric cancer. Ther Adv Med Oncol. 2019;11:1758835919874651.

18. Chen JL, Lin ZX, Qin YS, She YQ, Chen Y, Chen C, Qiu GD, Zheng JT, Chen ZL, Zhang SY. Overexpression of long noncoding RNA LINC01419 in esophageal squamous cell carcinoma and its relation to the sensitivity to 5-fluorouracil by mediating GSTP1 methylation. Ther Adv Med Oncol. 2019;11:1758835919838958.

19. Cheng Z, Hou S, Wu Y, Wang X, Sun Y, Liu B, Yuan M. LINC01419 promotes cell proliferation and metastasis in lung adenocarcinoma via sponging miR-519b-3p to up-regulate RCCD1. Biochem Biophys Res Commun. 2019;520(1):107-14

20. Yang Y, Chen D, Liu H, Yang K. Increased expression of IncRNA CASC9 promotes tumor progression by suppressing autophagy-mediated cell apoptosis via the AKT/mTOR pathway in oral squamous cell carcinoma. Cell Death Dis. 2019;10(2):41.

21. Tay Y, Kats L, Salmena L, Weiss D, Tan SM, Ala U, Karreth F, Poliseno L, Provero P, Di Cunto F, et al. Coding-independent regulation of the tumor suppressor PTEN by competing endogenous mRNAs. Cell. 2011;147(2):344-57

22. Salmena $L$, Poliseno $L$, Tay $Y$, Kats $L$, Pandolfi PP. A ceRNA hypothesis: the Rosetta Stone of a hidden RNA language? Cell. 2011;146(3):353-8.

23. Kager L, Tamamyan G, Bielack S. Novel insights and therapeutic interventions for pediatric osteosarcoma. Future Oncol (London, England). 2017;13(4):357-68. 
24. Dhamija S, Diederichs S. From junk to master regulators of invasion: IncRNA functions in migration, EMT and metastasis. Int J Cancer. 2016;139(2):269-80

25. Ai B, Kong X, Wang X, Zhang K, Yang X, Zhai J, Gao R, Qi Y, Wang J, Wang Z, et al. LINC01355 suppresses breast cancer growth through FOXO3-mediated transcriptional repression of CCND1. Cell Death Dis. 2019;10(7):502.

26. Guan H, Zhu T, Wu S, Liu S, Liu B, Wu J, Cai J, Zhu X, Zhang X, Zeng M, et al. Long noncoding RNA LINC00673-v4 promotes aggressiveness of lung adenocarcinoma via activating WNT/beta-catenin signaling. Proc Natl Acad Sci USA. 2019;116(28):14019-28.

27. Fei D, Zhang X, Liu J, Tan L, Xing J, Zhao D, Zhang Y. Long noncoding RNA FER1 L4 suppresses tumorigenesis by regulating the expression of PTEN targeting miR-18a-5p in osteosarcoma. Cell Physiol Biochem. 2018;51(3):1364-75.

28. Li Z, Wang Y, Hu R, Xu R, Xu W. LncRNA B4GALT1-AS1 recruits HuR to promote osteosarcoma cells stemness and migration via enhancing YAP transcriptional activity. Cell Prolif. 2018;51(6):e12504.

29. Zheng Y, Liu L, Shukla GC. A comprehensive review of web-based noncoding RNA resources for cancer research. Cancer Lett. 2017:407:1-8.

30. Zhang G, Pian C, Chen Z, Zhang J, Xu M, Zhang L, Chen Y. Identification of cancer-related miRNA-IncRNA biomarkers using a basic miRNA-IncRNA network. PLoS ONE. 2018;13(5):e0196681.

31. Zhang Y, Xu Y, Feng L, Li F, Sun Z, Wu T, Shi X, Li J, Li X. Comprehensive characterization of IncRNA-mRNA related ceRNA network across 12 major cancers. Oncotarget. 2016;7(39):64148-67.
32. Zhou M, Wang X, Shi H, Cheng L, Wang Z, Zhao H, Yang L, Sun J. Characterization of long non-coding RNA-associated ceRNA network to reveal potential prognostic IncRNA biomarkers in human ovarian cancer. Oncotarget. 2016;7(11):12598-611.

33. Pan R, He Z, Ruan W, Li S, Chen H, Chen Z, Liu F, Tian X, Nie Y. IncRNA FBXL19-AS1 regulates osteosarcoma cell proliferation, migration and invasion by sponging miR-346. OncoTargets Ther. 2018:11:8409-20.

34. Tan $\mathrm{H}$, Zhao L. IncRNA nuclear-enriched abundant transcript 1 promotes cell proliferation and invasion by targeting miR-186-5p/HIF-1alpha in osteosarcoma. J Cell Biochem. 2019;120(4):6502-14.

35. Tao Z, Chen S, Mao G, Xia H, Huang H, Ma H. The PDRG1 is an oncogene in lung cancer cells, promoting radioresistance via the ATM-P53 signaling pathway. Biomed Pharmacother. 2016:83:1471-7.

36. Zhang YJ, Li JQ, Li HZ, Song H, Wei CS, Zhang SQ. PDRG1 gene silencing contributes to inhibit the growth and induce apoptosis of gastric cancer cells. Pathol Res Pract. 2019;215(10):152567.

\section{Publisher's Note}

Springer Nature remains neutral with regard to jurisdictional claims in published maps and institutional affiliations.
Ready to submit your research? Choose BMC and benefit from:

- fast, convenient online submission

- thorough peer review by experienced researchers in your field

- rapid publication on acceptance

- support for research data, including large and complex data types

- gold Open Access which fosters wider collaboration and increased citations

- maximum visibility for your research: over $100 \mathrm{M}$ website views per year

At BMC, research is always in progress.

Learn more biomedcentral.com/submissions 